\title{
Optimal Control of a Class of One-Dimensional Nonlinear Distributed Parameter Systems with Discrete Actuators
}

\author{
Radhakant Padhi and S. N. Balakrishnan
}

\begin{abstract}
Combining the principles of dynamic inversion and optimization theory, a new approach is presented for stable control of a class of one-dimensional nonlinear distributed parameter systems with a finite number of actuators in the spatial domain. Unlike the existing 'approximate-then-design' and 'design-then-approximate' techniques, this approach does not use any approximation either of the system dynamics or of the resulting controller. The formulation has more practical significance because one can implement a set of discrete controllers with relative ease. To demonstrate the potential of the proposed technique, a reallife temperature control problem for a heat transfer application is solved through simulations. Numerical results are presented which show that the desired temperature profile can be achieved starting from any initial temperature profile.
\end{abstract}

\section{INTRODUCTION}

$\mathrm{C}$ ONTROL of distributed parameter systems has been studied from mathematical and engineering points of view. An interesting brief historical perspective of control of such systems can be found in [12]. There exist infinite-dimensional operator theory based methods for the control of distributed parameter systems. While there are many advantages, these operator theory based approaches are mainly limited to linear systems [9] and some limited class of problems like spatially invariant systems [3]. Moreover, for implementation purpose the infinitedimensional control solution needs to be approximated (e.g. truncating an infinite series, reducing the size of feedback gain matrix etc.) and hence is not completely free from errors. Such a control design approach is known as "designthen-approximate".

Another control design approach is "approximate-thendesign". Here, the PDEs describing the system dynamics are first approximated to yield a finite dimensional approximate model. This approximate system is then used for controller synthesis. In this approach, it is relatively easy to design controllers using various concepts of finitedimensional control design. An interested reader can refer

Radhakant Padhi, Asst. Professor, is with the Department of Aerospace Engineering, Indian Institute of Science, Bangalore, 560012, India (phone: +91-80-2293-2756, e-mail: padhi@ aero.iisc.ernet.in ).

S. N. Balakrishnan, Professor, is with the Department of Mechanical and Aerospace Engineering, University of Missouri - Rolla, MO, USA (phone: 573-341-4675, e-mail: bala@umr.edu ). to [ ${ }^{\mathrm{a}}$ Burns] for discussions on the relative merits and limitations of the two approaches.

An "approximate-then-design" approach to deal with the infinite dimensional systems is to have a finite dimensional approximation of the system using a set of orthogonal basis functions via Galerkin projection [11]. This technique normally leads to high order lumped system representations to adequately represent the properties of the original system, if arbitrary orthogonal functions are used as the basis functions. For this reason, in the recent literature attention is being increasingly given to new approximations. One powerful technique is called Proper Orthogonal Decomposition (POD). Out of numerous papers published on this topic and its use in control system design (both for linear and nonlinear DPS), we cite [4], [6]-[8], [11], [15][16] for reference. There are a few important drawbacks in the POD approach: (i) the technique is problem dependent and not generic; (ii) there is no guarantee that the snapshots will capture all dominant modes of the system and, more important, (iii) it is very difficult to have a set of 'good' snap-shot solutions for the closed-loop system prior to the control design. This is a serious limiting factor if one has to apply this technique for the closed-loop control design. Because of this reason, some attempts are being made in recent literature to adaptively redesign the basis functions, and hence the controller, in an iterative manner. An interested reader can see [1]-[2], [6] for a few ideas in this regard.

Even though the "design-then-approximate" and "approximate-then-design" approaches have been used in practice for designing the controllers for DPS, and there are attempts being made to generalize and refine these techniques, it will be nice to have a method which is independent of any such approximation, and hence, will fundamentally be different. The main goal of this paper is to present such an approach, which is applicable for a class of one-dimensional nonlinear distributed parameter systems. This has been done by combining the ideas of dynamic inversion [10], [13], [17] and optimization theory [5]. The formulation, which assumes a number of discrete controllers in the spatial domain, has more practical significance because one can implement a set of discrete controllers with relative ease (as compared to a continuous actuator). To demonstrate the potential of the proposed techniques, a real-life temperature control problem for a 
heat transfer application is solved. Numerical results from the simulations show that this method has great potential..

\section{PROBLEM DESCRIPTION}

\section{A. System Dynamics}

For control synthesis, a set of discrete controllers $\bar{u}_{m}$ that are located at $y_{m}(m=1, \cdots, M)$ are assumed. Assuming the width of the controller at $y_{m}$ to be $w_{m}$, the control action $\bar{u}_{m}$ satisfy the following properties:

- In the interval $\left[y_{m}-w_{m} / 2, y_{m}+w_{m} / 2\right]$, the controller $\bar{u}_{m}(t, y)$ is assumed to have a constant magnitude. Outside this interval, $\bar{u}_{m}=0$. Note that the interval $w_{m}$ may or may not be small.

- There is no overlapping of the controller located at $y_{m}$ with its neighboring controllers.

- No controller is placed exactly at the boundary. This assumption eliminates situations where control enters the system dynamics through boundary actions.

The system dynamics can now be written as follows:

$$
\dot{x}=f\left(x, x^{\prime}, x^{\prime \prime}, \cdots\right)+\sum_{m=1}^{M} g\left(x, x^{\prime}, x^{\prime \prime}, \cdots\right) \bar{u}_{m}
$$

where the state $x(t, y)$ is continuous functions of time $t \geq 0$ and spatial variable $y \in[0, L] . \dot{x}$ represents $\partial x / \partial t$ and $x^{\prime}, x^{\prime \prime}$ represent $\partial x / \partial y, \partial^{2} x / \partial y^{2}$ respectively. It is assumed that appropriate boundary conditions (e.g. Dirichlet, Neumann etc.) are available to make the system dynamics description Eq.(1) complete. The control variable appears linearly, and hence, the system dynamics is in the control affine form. Another assumption is that $g\left(x, x^{\prime}, x^{\prime \prime}, \ldots\right) \neq 0 \quad \forall t, y$.

\section{B. Goal for the Control Design}

The goal of the proposed controller is to ensure that the state variable $x(t, y) \rightarrow x^{*}(t, y)$ as $t \rightarrow \infty$ for all $y \in[0, L]$, where $x^{*}(t, y)$ is a known (possibly time-varying) profile in the domain $[0, L]$, which is continuous in $y$ and satisfies the spatial boundary conditions. Note that $x^{*}(t, y)$ satisfying the boundary conditions simplifies our task. This is because our discussion in this paper is limited to the class of problems where we do not have the control action at the boundary, and hence, it will be difficult to guarantee $x(t, y) \rightarrow x^{*}(y)$ at the boundary unless $x^{*}(y)$ itself satisfies the boundary condition.

\section{SYNTHESIS OF THE CONTROLLERS}

First, let us define an output (an integral error) as follows:

$$
z(t)=\frac{1}{2} \int_{0}^{L}\left[x(t, y)-x^{*}(t, y)\right]^{2} d y
$$

Note that when $z(t) \rightarrow 0, x(t, y) \rightarrow x^{*}(t, y)$ everywhere in $y \in[0, L]$. Next, the principle of dynamic inversion [10], [13], [17] is used to design a controller such that the following first-order equation is satisfied:

$$
\dot{z}+k z=0
$$

where $k>0$ serves as a gain; an appropriate value of $\mathrm{k}$ has to be chosen by the control designer. For a better physical interpretation, one may choose it as $k=(1 / \tau)$, where $\tau>0$ serves as a "time constant" for the error $z(t)$ to decay. Using the definition of $z$ in Eq.(2), Eq.(3) leads to

$$
\int_{0}^{L}\left(x-x^{*}\right)\left(\dot{x}-\dot{x}^{*}\right) d y=-\frac{k}{2} \int_{0}^{L}\left(x-x^{*}\right)^{2} d y
$$

Substituting for $\dot{x}$ from Eq.(1) in Eq.(4) and simplifying we arrive at:

$$
\int_{0}^{L}\left(x-x^{*}\right) g\left(x, x^{\prime}, x^{\prime \prime}, \ldots\right) u d y=\gamma
$$

where

$$
\gamma \triangleq-\int_{0}^{L}\left(x-x^{*}\right)\left[f\left(x, x^{\prime}, x^{\prime \prime}, \ldots\right)-\dot{x}^{*}\right] d y-\frac{k}{2} \int_{0}^{L}\left(x-x^{*}\right)^{2} d y
$$

Expanding Eq.(5a), we can write

$$
\left[\int_{y_{1}-\frac{-}{2}}^{y_{1}+\frac{w_{1}}{2}}\left(x-x^{*}\right) g d y\right] \bar{u}_{1}+\cdots+\left[\int_{y_{M}}^{y_{M}+\frac{w_{M}}{2}}\left(x-x^{*}\right) g d y\right] \bar{u}_{M}=\gamma
$$

For convenience, we define

$$
I_{m} \triangleq \int_{y_{m}-\frac{w_{m}}{2}}^{y_{m}+\frac{w_{m}}{2}}\left(x-x^{*}\right) g d y, \quad m=1, \ldots, M
$$

Then from Eqs.(6) and (7), we can write

$$
I_{1} \bar{u}_{1}+\cdots+I_{M} \bar{u}_{M}=\gamma
$$

Eq.(8) will guarantee that eventually $z(t) \rightarrow 0$ as $t \rightarrow \infty$. However, note that Eq.(8) is a single equation with $M$ variables $u_{m}, m=1, \ldots, M$ and hence we have infinitely many solutions. In order to obtain a unique solution, we formulate an optimal control problem that that will have Eq.(8) as a control while minimizing the following cost function

$$
J=\frac{1}{2}\left(r_{1} w_{1} \bar{u}_{1}^{2}+\cdots+r_{m} w_{m} \bar{u}_{m}^{2}\right)
$$

In other words, we wish to minimize the cost function in Eq.(9), subjected to the constraint in Eq.(8). It can be seen that that the resulting solutions will lead to a minimum control effort. In Eq.(9), choosing appropriate values for $r_{1}, \ldots, r_{m}>0$ gives a control designer the flexibility of putting relative importance of the control magnitude at different spatial locations $y_{m}, m=1, \ldots, M$.

To use techniques of constrained optimization [5], we first formulate the following augmented cost function

$$
\bar{J}=\frac{1}{2}\left(r_{1} w_{1} \bar{u}_{1}^{2}+\cdots+r_{m} w_{m} \bar{u}_{m}^{2}\right)+\lambda\left[\left(I_{1} \bar{u}_{1}+\ldots+I_{m} \bar{u}_{m}\right)-\gamma\right]
$$

where $\lambda$ is a Lagrange multiplier, which is a free variable needed to convert the constrained optimization problem to a free optimization problem. In Eq.(10), $\lambda$ and 
$u_{m}, m=1, \ldots, M$ are free variables, with respect to which the minimization has to be carried out.

The necessary condition of optimality is given by [Bryson]

$$
\begin{aligned}
& \frac{\partial \bar{J}}{\partial u_{m}}=0, \quad m=1, \ldots, M \\
& \frac{\partial \bar{J}}{\partial \lambda}=0
\end{aligned}
$$

Expanding Eqs.(11) and (12) leads to:

$$
\begin{aligned}
& r_{m} w_{m} \bar{u}_{m}+I_{m} \lambda=0, \quad m=1, \ldots, M \\
& I_{1} \bar{u}_{1}+\cdots+I_{M} \bar{u}_{M}=\gamma
\end{aligned}
$$

Solving for $\bar{u}_{1}, \ldots, \bar{u}_{M}$ from Eq.(13), substituting those in Eq.(14) and solving for $\lambda$ we get

$$
\lambda=-\gamma / \sum_{m=1}^{M}\left[I_{m}^{2} /\left(r_{m} w_{m}\right)\right]
$$

Eqs.(13) and (15) lead to the following expression

$$
\bar{u}_{m}=I_{m} \gamma /\left[r_{m} w_{m} \sum_{\tilde{m}=1}^{M} I_{\tilde{m}}^{2} /\left(r_{\tilde{m}} w_{\tilde{m}}\right)\right], \quad m=1, \ldots, M
$$

As a special case, when $r_{1}=\cdots=r_{M}$ (i.e. equal importance is given for minimization of all controllers) and $w_{1}=\cdots=w_{m}$ (i.e. widths of all controllers are same), we have

$$
\bar{u}_{m}=I_{m} \gamma /\left(\|I\|_{2}\right)^{2}
$$

where $I \triangleq\left[\begin{array}{lll}I_{1} & \cdots & I_{M}\end{array}\right]^{T}$. Note that in cases where a number of controllers are being used over different control application widths (i.e. $u_{m}, m=1, \ldots, M$ are different), we can still use the simplified formula in Eq.(17), by selecting $r_{1}, \cdots, r_{M}$ such that $r_{1} w_{1}=\cdots=r_{M} w_{M}$.

\section{Singularity in Control Solution and Revised Goal:}

From Eqs.(16) and (17), it is clear that when $\|I\|_{2}^{2} \rightarrow 0$ (which happens when all of $I_{1}, \ldots, I_{M} \rightarrow 0$ ) and $\gamma \succ 0$, we have the problem of control singularity in the sense that $\bar{u}_{m} \rightarrow \infty$ (since the denominators of Eqs.(16) and (17) go to zero faster than the corresponding numerators). Note that if the number of controllers $M$ is large, probably the occurrence of such a singularity is a rare possibility, since all of $I_{1}, \ldots, I_{M} \rightarrow 0$ simultaneously is a strong condition.

Nonetheless such a case may arise in transition. More important, this issue of control singularity will always arise when $x(t, y) \rightarrow x^{*}(t, y), \forall y \in[0, L]$ (which is the primary goal of the control design). This happens probably because we have only limited control authority (controllers are available only in a subset of the spatial domain), where as we have aimed to achieve a much bigger goal of tracking the state profile $\forall y \in[0, L]$ - something that is beyond the capability of the controllers. Hence whenever such a case arises (i.e. when all of $I_{1}, \ldots, I_{M} \rightarrow 0$ or, equivalently, $\|I\|_{2} \rightarrow 0$ ), to avoid the issue of control singularity, we propose to redefine the goal as follows.
First, define $X \triangleq\left[x_{1}, \cdots, x_{M}\right]^{T}, X^{*} \triangleq\left[x_{1}^{*}, \cdots, x_{M}^{*}\right]^{T}$ and the error vector $E \triangleq\left(X-X^{*}\right)$. Next, design a controller such that $E \rightarrow 0$ as $t \rightarrow \infty$. In other words, the aim is to guarantee that the values of the state variable at the node points $\left(y_{m}, \quad m=1, \ldots, M\right)$ track their corresponding desired values. To accomplish this goal, select a positive definite gain matrix $K$ such that:

$$
\dot{E}+K E=0
$$

One way of selecting such a gain matrix $K$ is to choose it a diagonal matrix with $m^{\text {th }}$ diagonal element being $k_{m}=\left(1 / \tau_{m}\right)$ where $\tau_{m}>0$ is the desired time constant of the error dynamics. In such a case, the $m^{\text {th }}$ channel of Eq.(18) can be written as

$$
\dot{e}_{m}+k_{m} e_{m}=0
$$

Expanding the expressions for $e_{m}$ and $\dot{e}_{m}$ and solving for $\bar{u}_{m}(m=1, \ldots, M)$, we obtain

$$
\bar{u}_{m}=\frac{1}{g_{m}}\left[\dot{x}_{m}^{*}-f_{m}-k_{m}\left(x_{m}-x_{m}^{*}\right)\right]
$$

where

$x_{m} \triangleq x\left(t, y_{m}\right), x_{m}^{*} \triangleq x^{*}\left(t, y_{m}\right), f_{m} \triangleq f\left(t, y_{m}\right), g_{m} \triangleq g\left(t, y_{m}\right)$

\section{Final Control Solution for Implementation}

Combining the results in Eqs.(16) and (20a), we finally write the control solution as

$$
\bar{u}_{m}=\left\{\begin{array}{ll}
\frac{1}{g_{m}}\left[\dot{x}_{m}^{*}-f_{m}-k_{m}\left(x_{m}-x_{m}^{*}\right)\right], & \text { if }\|I\|_{2}<\text { tol } \\
\left(I_{m} \gamma\right) /\left(r_{m} w_{m} \sum_{\tilde{m}=1}^{M} I_{\tilde{m}}^{2} /\left(r_{\tilde{m}} w_{\tilde{m}}\right)\right), & \text { otherwise }
\end{array}\right\}
$$

where tol represents a tolerance value. An appropriate value for it can be fixed by the control designer (a convenient way to fix a good value for it is from trial-anderror simulation studies). Note that some discontinuity/jump in control magnitude is expected when switching takes place. However, this jump can be minimized by selecting a sufficiently low value for tol within achievable limits of the control magnitude. Moreover, this behavior will be further reduced by considering the actuator dynamics (not considered here).

\section{A MOTIVATING NONLINEAR PROBLEM}

\section{A. Mathematical Model}

The problem used to demonstrate the theories presented in Section III is a real-life problem. It involves the heat transfer in a fin of a heat exchanger, as depicted in Figure 2. 


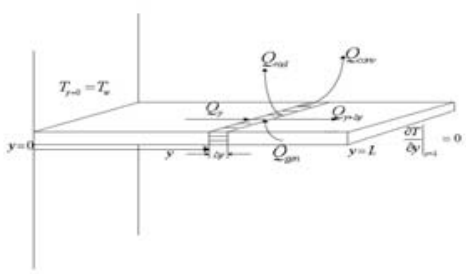

Figure 2: Schematic of the physics of the problem

First, a mathematical model is developed from the principles of heat transfer [14]. Using the law of conservation of energy in an infinitesimal volume at a distance $y$ having a length $\Delta y$,

$$
Q_{y}+Q_{g e n}=Q_{y+\Delta y}+Q_{c o n v}+Q_{r a d}+Q_{c h g}
$$

where $Q_{y}$ is the rate of heat conducted in, $Q_{\text {gen }}$ is the rate of heat generated, $Q_{y+\Delta y}$ is the rate of heat conducted out, $Q_{\text {conv }}$ is the rate of heat convected out, $Q_{\text {rad }}$ is the rate of heat radiated out and $Q_{c h g}$ is the rate of heat change. Next, from the laws of physics for heat transfer [14], the following expressions can be obtained

$$
\begin{aligned}
& Q_{y}=-k A(\partial T / \partial y) \\
& Q_{g e n}=S A \Delta y \\
& Q_{\text {conv }}=h P \Delta y\left(T-T_{\infty_{1}}\right) \\
& Q_{\text {rad }}=\varepsilon \sigma P \Delta y\left(T^{4}-T_{\infty_{2}}^{4}\right) \\
& Q_{\text {chg }}=\rho C A \Delta y(\partial T / \partial t)
\end{aligned}
$$

In Eqs.(23a-e), $T(t, y)$ represents the temperature (this is the state $x(t, y)$ in the context of discussion in Section III), which is a function of both time $t$ and spatial location $y$. $S(t, y)$ is the rate of heat generation per unit volume (this is the control $u$ in the context of discussion in Section 3 ) for this problem. The meanings of various parameters and their numerical values used and are given in Table 1.

Table 1: Definitions and numerical values of the parameters

\begin{tabular}{|l|l|l|}
\hline Parameter & Meaning & Numerical value \\
\hline$k$ & Thermal conductivity & $180 \mathrm{~W} /\left(\mathrm{m}^{\circ} \mathrm{C}\right)$ \\
\hline$A$ & Cross sectional area & $2 \mathrm{~cm}^{2}$ \\
\hline$P$ & Perimeter & $9 \mathrm{~cm}$ \\
\hline$h$ & $\begin{array}{l}\text { Convective heat transfer } \\
\text { coefficient }\end{array}$ & $5 \mathrm{~W} /\left(\mathrm{m}^{2}{ }^{\circ} \mathrm{C}\right)$ \\
\hline$T_{\infty_{1}}$ & $\begin{array}{l}\text { Temperature of the medium } \\
\text { in the immediate } \\
\text { surrounding of the surface }\end{array}$ & $30^{\circ} \mathrm{C}$ \\
\hline$T_{\infty_{2}}$ & $\begin{array}{l}\text { Temperature at a far away } \\
\text { place in the direction normal } \\
\text { to the surface }\end{array}$ & $-40^{\circ} \mathrm{C}$ \\
\hline
\end{tabular}

\begin{tabular}{|l|l|l|}
\hline$\epsilon$ & Emissivity of the material & 0.2 \\
\hline$\sigma$ & Stefan-Boltzmann constant & $5.669 \times 10^{-8} \mathrm{~W} / \mathrm{m}^{2} \mathrm{~K}^{4}$ \\
\hline$\rho$ & Density of the material & $2700 \mathrm{~kg} / \mathrm{m}^{3}$ \\
\hline$C$ & Specific heat of the material & $860 \mathrm{~J} /\left(\mathrm{kg}^{\circ} \mathrm{C}\right)$ \\
\hline
\end{tabular}

These representative values are valid for Aluminum. The area $A$ and perimeter $P$ have been computed assuming a fin of dimension $40 \mathrm{~cm} \times 4 \mathrm{~cm} \times 0.5 \mathrm{~cm}$. Note that a onedimensional approximation has been assumed which means that uniform temperatures in the other two dimensions are assumed to be arrived at instantaneously.

Using Taylor series expansion and considering a small $\Delta y \rightarrow 0$, we can write

$$
Q_{y+\Delta y} \approx Q_{y}+\left(\frac{\partial Q_{y}}{\partial y}\right) \Delta y
$$

Using Eqs.(23a-e) and (24) in Eq.(22) and simplifying, we can write

$$
\frac{\partial T}{\partial t}=\frac{k}{\rho C}\left(\frac{\partial^{2} T}{\partial y^{2}}\right)-\frac{P}{A \rho C}\left[h\left(T-T_{\infty_{1}}\right)+\varepsilon \sigma\left(T^{4}-T_{\infty_{2}}^{4}\right)\right]+\left(\frac{1}{\rho C}\right) S
$$

For convenience, define $\alpha_{1} \triangleq(k / \rho C), \alpha_{2} \triangleq-(P h) /(A \rho C)$, $\alpha_{3} \triangleq-(P \varepsilon \sigma) /(A \rho C)$ and $\beta \triangleq 1 /(\rho C)$, Eq.(25) can be rewritten as

$$
\frac{\partial T}{\partial t}=\alpha_{1}\left(\frac{\partial^{2} T}{\partial y^{2}}\right)+\alpha_{2}\left(T-T_{\infty_{1}}\right)+\alpha_{3}\left(T^{4}-T_{\infty_{2}}^{4}\right)+\beta S
$$

However, since the source (control) is not present for the entire spatial domain, $S$ is not a continuous function of $y$. There is a discrete set of controllers, located at $\left[y_{m}-w_{m} / 2, y_{m}+w_{m} / 2\right], \quad m=1, \ldots, M$. Further, by our assumption, the controller has a constant magnitude $\bar{S}_{m}$ in the interval $\left[y_{m}-w_{m} / 2, y_{m}+w_{m} / 2\right]$. Because of this, the system dynamics is given by:

$$
\frac{\partial T}{\partial t}=\alpha_{1}\left(\frac{\partial^{2} T}{\partial y^{2}}\right)+\alpha_{2}\left(T-T_{\infty_{1}}\right)+\alpha_{3}\left(T^{4}-T_{\infty_{2}}^{4}\right)+\beta \sum_{m=1}^{M} \bar{S}_{m}
$$

Along with Eq.(26), the following boundary conditions are assumed.

$$
T_{y=0}=T_{w},\left.\quad(\partial T / \partial y)\right|_{y=L}=0
$$

where $T_{w}$ is the wall temperature. Insulated boundary condition at the tip is assumed with the assumption that either there is some physical insulation at the tip or heat loss at the tip due to convection and radiation is negligible (mainly because of its low surface area). The goal of the controller was to make sure that the actual temperature profile $T(t, y) \rightarrow T^{*}(y)$, where $T^{*}(y)$ was chosen to be a constant (with respect to time) temperature profile given by the following expression

$$
T^{*}(y)=T_{w}+\left(T_{w}-T_{\text {tip }}\right)^{-\zeta y}
$$


where we selected the wall temperature $T_{w}=150{ }^{\circ} \mathrm{C}$, fin tip temperature $T_{t i p}=130{ }^{\circ} \mathrm{C}$ and the decaying parameter $\zeta=20$. The selection such a $T^{*}(y)$ from Eq.(29) was motivated by the fact that it leads to a smooth continuous temperature profile across the spatial dimension $y$. Note that this $T^{*}(y)$ satisfies the boundary condition at $y=0$ exactly and at $y=L$ approximately, with a very small (rather negligible) approximation error (because of the selection for a high value of $\zeta$ ). Note that the system dynamics is in control-affine form and $g\left(x, x^{\prime}, x^{\prime \prime}, \cdots\right)=\beta$, a constant number that is not equal to zero. Moreover, there is no boundary control action. This is compatible with the class of DPS for which control synthesis theories were developed in Section III.

In our simulation studies, the control gain was set as $k=(1 / \tau), \quad$ where $\tau=30 \mathrm{sec}$ and the tolerance value tol $=0.001$. After switching, the control gain $K=\operatorname{diag}\left(k_{1} \cdots \cdots k_{M}\right)$ was used and selected $k_{m}=1 / \tau_{m}$, $\tau_{m}=\tau$ for $m=1, \ldots, M$. The parameters $w_{1}=\cdots=w_{M}=2 \mathrm{~cm}$ and it was assumed $r_{1}=\cdots=r_{M}$. With this assumption, we could use the simplified expression for the controller (Eq.(17)) whenever $\|I\|_{2}>$ tol and did not need numerical values for $r_{1}, \cdots, r_{M}$. To begin with, five $(M=5)$ equally spaced controllers were used.

\section{B. Analysis of Numerical Results}

First an initial condition (profile) for the temperature was obtained from the expression $T(0, y)=T_{m}+x(0, y)$, where $T_{m}=150{ }^{\circ} \mathrm{C} \quad$ (a constant value) serves as the mean temperature and $x(0, y)$ represents the deviation from $T_{m}$. Taking $A=50$ we computed $x(0, y)$ as $x(0, y)=(A / 2)+(A / 2) \cos (-\pi+2 \pi y / L)$. Applying the controller as synthesized in Eq.(21), the system was simulated as given in Eqs.(27-28) from time $t=t_{0}=0$ to $t=t_{f}=5 \mathrm{~min}$. The simulation results obtained are presented in Figures 3-4. Note from Figure 3 that the goal of tracking $T^{*}(y)$ is roughly met. The associated control (rate of energy input) profile $S(t, y)$ obtained is as shown in Figure 4. The figure shows that the required control magnitude to achieve the objective is not high, in the entire spatial domain $[0, L]$ and for all time $t \in\left[t_{0}, t_{f}\right]$.

There are also some concerns evident from Figures 3-4. First, there are small jumps in the control histories when the control switching takes place (at about $2.5 \mathrm{~min}$ ). Moreover, there is a weaving pattern to the state profile as $T(t, y) \rightarrow T^{*}(y)$, and hence, the goal of the control design is not met fully. Both of these probably happened because we assumed a small number of discrete controllers. As pointed out in Section III, one way of minimizing this effect is to increase the number of controllers. Hence, next we selected ten controllers (instead of five) and carried out the simulation again. The results are shown in Figures 5-6.

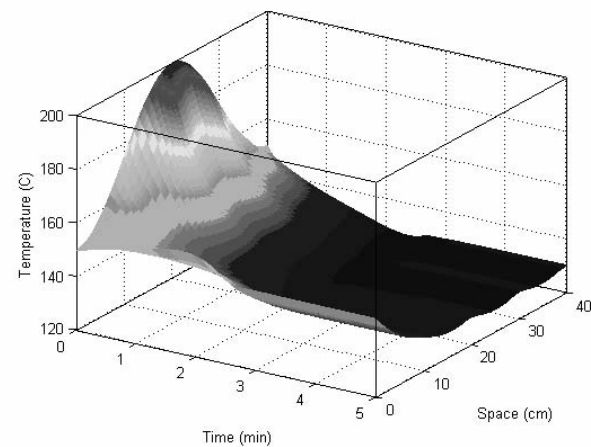

Figure 3: Evolution of the temperature (state) profile from a sinusoidal initial condition

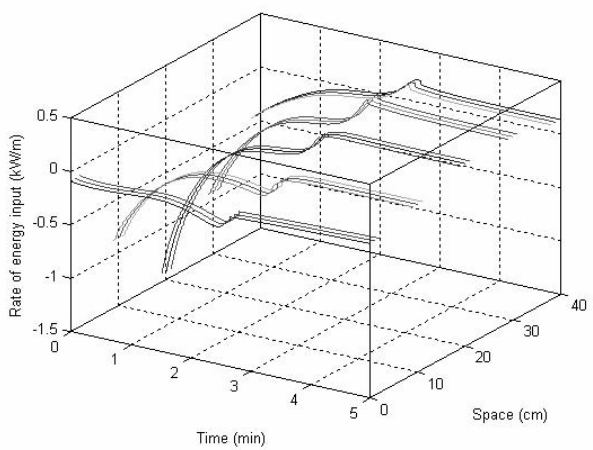

Figure 4: Rate of energy inputs (controllers) for the evolution of temperature profile in Figure 4

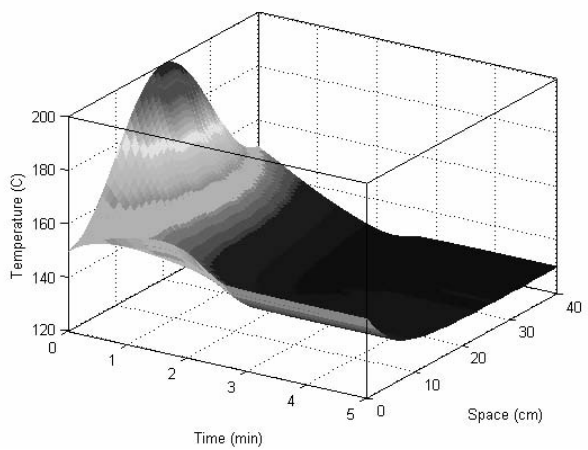

Figure 5: Evolution of the temperature (state) profile from a sinusoidal initial condition 


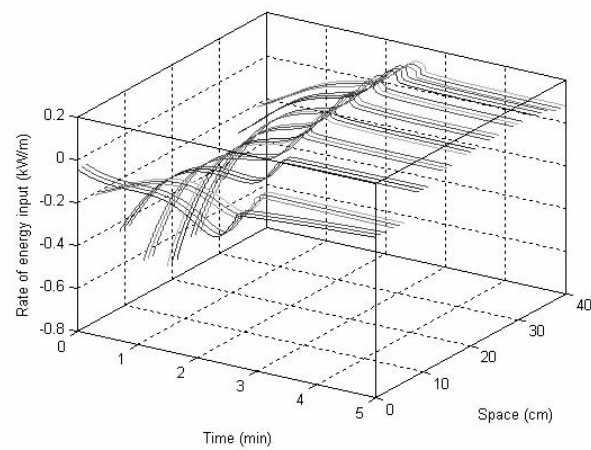

Figure 6: Rate of energy inputs (controllers) for the evolution of temperature profile in Figure 7

It is quite clear from Figures 5-6 that the weaving nature is substantially smaller and the goal $T(t, y) \rightarrow T^{*}(y), \forall y \in[0, L]$ is met more accurately.

Moreover, note that as compared to the case with five controllers, the control effectiveness now is higher a fact which leads to smaller magnitudes of the controllers (compare Figures 4 and 6).

To demonstrate the generality of this technique, a number of random profiles for $T(0, y)$ were considered. A number of random profiles were generated using the relationship $T(0, y)=T_{m}+x(0, y)$, where $x(0, y)$ came through a Fourier

Series, such that it satisfies $\|x(0, y)\|^{2} \leq k_{1}\|x\|_{\max }^{2}$, $\left\|x^{\prime}(0, y)\right\|^{2} \leq k_{2}\left\|x^{\prime}\right\|_{\max }^{2}$ and $\left\|x^{\prime \prime}(0, y)\right\|^{2} \leq k_{3}\left\|x^{\prime \prime}\right\|_{\max }^{2}$. The values for $\|x\|_{\max },\left\|x^{\prime}\right\|_{\max }$ and $\left\|x^{\prime \prime}\right\|_{\max }$ were computed using an envelope profile $x_{e n v}(y)=A \sin (\pi y / L)$. The norm used is the $L_{2}$ norm defined by $\|x\| \triangleq\left(\int_{0}^{L} x^{2}(y) d y\right)^{1 / 2}$. The value of parameter $A$ was set at 50 with $k_{1}=2, k_{2}=k_{3}=10$. For more details about the generation of these random profiles, the reader is referred to [15]. A large number of random initial conditions (state profiles) were led to results very similar to ones presented in Figures 3-6 and are omitted here, for lack of space. However, this confirms to the claim that the method presented in this paper is independent of the initial condition and can be used for any arbitrary initial condition.

\section{CONCLUSIONS}

Principles of dynamic inversion and optimization theory, have been used to develop a fairly general control synthesis technique for a class of one-dimensional nonlinear distributed parameter systems. The formulation has good practical significance because one can implement a set of discrete controllers with relative ease (as compared to a continuous actuator). The technique presented in this paper can be implemented online(feedback), since we obtain closed form solution for the control variable.

\section{ACKNOWLEDGEMENT:}

This research was supported by NSF-USA grants 0201076 and 0324428. The authors wish thank Dr. D. C. Look, Emer.Professor, Dept. of Mechanical and Aerospace Engineering, University of Missouri-Rolla, USA, in connection with the real-life heat transfer problem.

\section{REFERENCES}

[1]Annaswamy A., Choi J. J., Sahoo D., Active Closed Loop Control of Supersonic Impinging Jet Flows Using POD Models, Pro.s of the $41^{\text {st }}$ IEEE Con. On Dec and Control, Las Vegas, 2002.

[2] Arian E., Fahl M. and Sachs E. W., Trust-region Proper Orthogonal Decomposition for Flow Control, NASA/CR-2000-210124, ICASE Report No. 2000-25.

[3] Bameih B., The Structure of Optimal Controllers of Spatiallyinvariant Distributed Parameter Systems. Proceedings of the Conference on Decision \& Control, 1997, 1056-1061.

[4] Banks H. T., Rosario R. C. H and Smith R. C., Reduced-Order Model Feedback Control Design: Numerical Implementation in a Thin Shell Model, IEEE Tran, on Auto. Cotn., Vol. 45, 2000.

[5] Bryson A. E. and Ho Y. C., Applied Optimal Control, London: Taylor and Francis, 1975.

[6] Burns J.A. and King, B.B., Optimal sensor location for robust control of distributed parameter systems. Proceedings of the Conference on Decision and Control, 1994, pp. 3967- 3972.

[7] Burns J. and King B. B., A Reduced Basis Approach to the Design of Low-order Feedback Controllers for Nonlinear Continuous Systems, Journal of Vibration and Control, Vol.4, 1998, 297-323.

[8] Christofides P. D., Nonlinear and Robust Control of PDE Systems Methods and Applications to Transport-Reaction Processes, Birkhäuser, Boston, 2000.

[9] Curtain R. F. and Zwart H. J., An Intro. to Infinite Dimensional Linear Systems Theory, Springer-Verlag, New York, 1995.

[10] Enns, D., Bugajski, D., Hendrick, R. and Stein, G., Dynamic Inversion: An Evolving Methodology for Flight Control Design, International Journal of Control, Vol.59, No.1,1994, pp.71-91.

[11] Holmes P., Lumley J. L. and Berkooz G., Turbulence, Coherent Structures, Dynamical Systems and Symmetry, Cambridge University Press, 1996, 87-154.

[12] Lasiecka I., Control of Systems Governed by Partial Differential Equations: A Historical Perspective, Proceedings of the $34^{\text {th }}$ Conference on Decision and control, 1995, 2792-2796.

[13] Lane, S. H. and Stengel, R. F., Flight Control Using Non-Linear Inverse Dynamics, Automatica, Vol.24, No.4, 1988, pp.471-483.

[14] Miller A. F., Basic Heat and Mass Transfer, Richard D. Irwin Inc., MA, 1995.

[15] Padhi R. and Balakrishnan S. N., Proper Orthogonal Decomposition Based Feedback Optimal Control Synthesis of Distributed Parameter Systems Using Neural Networks, Proceedings of the American Control Conference, 2002, pp. 4389-4394.

[16] Ravindran S. S., Adaptive Reduced-Order Controllers for a Thermal Flow System Using Proper Orthogonal Decomposition, SIAM Journal on Scientific Computing, Vol.23, No.6, 2002

[17] Slotine, J-J. E. and Li, W., Applied Nonlinear Control, Prentice Hall, 1991. 